\title{
Enhanced Crowd-Sourced Video Sharing System
}

\author{
Anuradha S. Kadam \\ Department Of Computer Engineering \\ MMCOE,Pune \\ SavitribaiPhule University, \\ Pune , India
}

\author{
Rahul V. Dagade \\ Department Of Computer Engineering \\ MMCOE,Pune \\ SavitribaiPhule University, \\ Pune, India
}

\begin{abstract}
Nowadays video capturing and sharing is common. Consider an event, meeting of Facebook founder and Prime minister of the India. Hundreds of people captured that meet in mobile phones and uploaded it to video sharing applications(VSA) like Twitter, Facebook. Huge amount of the bandwidth and battery is used by this activity of uploading videos of the same event. To overcome this problem existing systems used On-demand retrieval approach here first metadata of the video is uploaded to server. If query from user is matched with metadata stored at the server then video is fetch from smartphone of the user who uploaded the video. On-demand approach proved its effectiveness. Limitation of the approach is its speed due to after query of the user, video is extracted and then given to requester. To overcome this problem and inherit the advantages of the on-demand approach system proposed effective video sharing system for event with objective to reduce overlapping videos and time for fetching video.
\end{abstract}

\section{Keywords}

Crowd Source Video, Video Sharing, Spatio-Temporal Query.

\section{INTRODUCTION}

Integrated high quality cameras to the mobiles in cheaper rates and availability of internet resulted into video sharing trend. In many events where public present in large numbers, events is recorded by many people and shared with their friends. In many contexts, single user can not shoot whole event. Crowdsourcing is one approach which enables to get more complete coverage of the event.

In existing system video is uploaded on servers then it is accessible to the users. When users query matches with uploaded videos then that uploaded video is provided to the user. Current system uses keywords to retrieve required video. Some events are attended by thousands of the people. As the public increases number of captured videos records increases and indirectly number of share also increases.

What will be effect on event side?

All people are at the same location sharing the heavy data i.e. videos at the same time increases the load on the network results into degradation of quality network. User's mobile devices has limited battery, poor network may need more energy to upload the video.

What will be effect on the server side?

As all videos are uploaded of same event, there is high possibility of redundant videos.

Another problem of existing video sharing system is that if users want to see video of same event then keywords will be same. Given keyword will give hundreds of videos of the same event. But if user wants to see video of particular time then such query is not supported.

To avoid this problem paper [1] proposed novel system named as Movisode. Movisode stands for MObileVIdeo Sharing On-DEmand. This video sharing system is Event centric which assumes that if user is registered for some event he will upload videos of that event only. Movisode has the following features:

1) Support of Spatiotemporal query, in such queries user can specify the time, angle from which video is shoot and Point of interest (POI) of camera.

2) Videos are not uploaded directly to the server; metadata of the video is generated and metadata is uploaded to the server. If users query is matched with the metadata then that video is fetched from user who uploaded the metadata. This video sharing system works with On-demand feature.

Movisode system is composed of two core algorithms, one is for metadata extraction from video and another is for selection of video to be fetched for spatio temporal query of the user. Experimental results proved the effectiveness of the Movisode system.

\section{LITERATURE REVIEW \\ 1) Collaborative filtering}

MoVi [2] is designed to capture social events collaboratively using sensory cues as ambient sound and light to detect changes in environment.

CrowdOptics[3] facilitates browsing sensor annotated videos in crowded events.Micro-Cool is designed with aim to create the single video from many videos which are captured by the public in the event.

\section{2) Geo-Tagging based Techniques}

Event coverage and sharing also done by various geo tagging based techniques that uses in built sensors in Smart Phones Paper [4] discusses how a sensor-rich world can be used by digital recording devices to increase a user's ability to search through a large database of picture and videos. Paper describes the architecture and implements a digital recording system that captures identities and locations of things along with photos. Proposed system named as sensor-enhanced video annotation (SEVA), appends a series of correlation, interpolation, and extrapolation techniques. Tagged stream is generated that can be used to efficiently search for videos or images containing particular things or people.

Paper [5] addressed core issues in mobile surveillance and architecture for querying and retrieving distributed semipermanent multi-modal data through weak networks with limited connectivity. Paper provides [6] a rich set of queries for spatio-temporal querying for surveillance case. The magnitude of area or time interval captured in each video clip is used with sensor metadata to rank relevant video clips. After ranking they are filtered based on the Wiki Information. This work is improved in [7] to introduce energy conservation 
by uploading metadata on-demand, and by using WiFi/GSMbased location inference to complement GPS.

\section{3) Crowdsourced Video Retrieval}

GigaSight [8] filters videos on a cloud infrastructure, trying to identify privacy-sensitive information from crowdsourced video and uses it for retrieval from cloud. FOCUS [9] uses Structure-from-Motion (SfM) [10] as part of cloud-based architecture for organizing videos. SfM requires all videos to be available for frame-by-frame pairwise comparison of all videos, and camera calibration for all the cameras involved in the video capture.

\subsection{Motivation}

Existing system designed with the principle of On-demand retrieval of video from up-loaders smart phones for viewers. In existing system, when viewer submits his query q, metadata which matches with query $q$ is searched after that fetch request is send to the metadata up-loader and then on the availability of the up-loaders respective video is fetched. Once required video is fetched, it is given to viewer.

Above process takes large time to server the user. Time to serve the video to viewer depends on the availability of the metadata up-loader, network bandwidth and battery of the uploader's mobile. To overcome these problems there is need to design system which will serve the user with less time requirement and all advantages of the existing system

Table 1: Comparison of proposed and existing systems

\begin{tabular}{|c|c|c|}
\hline & \begin{tabular}{l}
\multicolumn{1}{c}{ On demand } \\
Retrieval of \\
Crowd-sourced \\
Mobile Video
\end{tabular} & \begin{tabular}{ll}
\multicolumn{2}{c}{ Enhanced } \\
Crowd-Sourced \\
Video $\quad$ Sharing \\
System
\end{tabular} \\
\hline Response time & High & Low \\
\hline \begin{tabular}{l}
\multicolumn{2}{c}{ Dependency on } \\
Bandwidth of \\
smartphone of user \\
which uploads \\
video after \\
receiving query
\end{tabular} & Yes & No \\
\hline \begin{tabular}{l}
\multicolumn{2}{c}{ Dependency on } \\
Battery of \\
smartphone of user \\
which uploads \\
video after \\
receiving query
\end{tabular} & Yes & No \\
\hline $\begin{array}{l}\text { All videos are } \\
\text { uploaded to server? }\end{array}$ & No & No \\
\hline $\begin{array}{l}\text { Video fetching } \\
\text { strategy }\end{array}$ & On demand & $\begin{array}{l}\text { Decision } \\
\text { system }\end{array}$ \\
\hline
\end{tabular}

\section{PROPOSED SYSTEM}

Figure 1 show the architecture of the proposed system and description of the system is as follows.

\section{1) Event}

Event manager will register the event to the video sharing system and also submit the reference image of the scene or stage of the event. Reference image will be used to evaluate the point of interest of the video.

\section{2) Users capture the videos}

When user reached to the event, user check-ins to the registered video. Check-in is optional because GPS is not available for indoor events. When event is started multiple users shoot the event with various duration, Point of Interest. Arrow 1 in fig 1 represents that video is captured by users. Once video is captured, metadata of the video is evaluated at the user's smartphones. Metadata contains Start time of the video, end time of the video, Point of Interest of the video.Point of Interest is as per given in paper [1]. Once metadata is calculated of the video, $\mathrm{M}$ is uploaded to the server (shown as arrow no 2 in fig 1). It is very important that metadata evaluation should be low computation task and size of the metadata should be lesser than actual video.

\section{3) System Server- System server contains two sub-blocks}

- Decision System - Once the metadata of the all videos from single event are received at System server. Decision system uses algorithm to select which videos to be fetched from the available metadata. As described in the algorithm First step is finding duplicate videos i.e. videos with same or overlapping duration and approximate same Point of Interest. Decision System sends request to upload the videos to users (arrow 3 in Fig 1). After fetch request videos are uploaded to the server.

- Retrieval Unit - User who wants to view the video sends the spatiotemporal query q to the video sharing system for particular event. Query may contain start time, end time, and Point of Interest. Point of Interest is optional part in the query for user's convenience. Viewer user query is shown as arrow A in Fig 1. Once query is received at Retrieval Unit, relevant video is retrieved by matching the start time, end time and Point of interest of videos and query. Retrieved videos are given to the user. (B arrow in Fig 1)

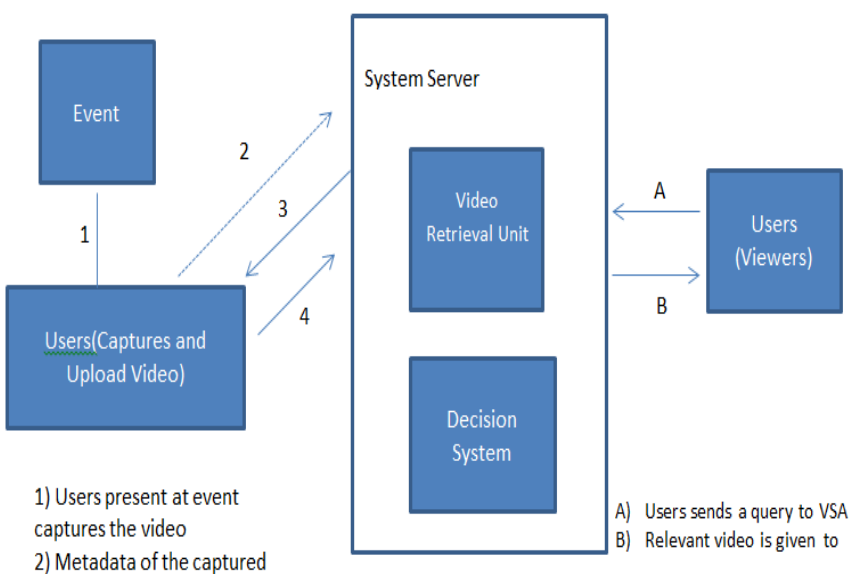

video is sent to VSA

3) Decision System in the VSA

takes decision and sends fetch

request to user if decision is yes

Fig 1.Video Sharing System for Event Coverage

\section{PROPOSED ALGORITHM}

In this work, system proposed an algorithm to get the list of videos needs to be extracted from user's mobiles. Input to this site will be metadata of the videos and Point of interest variation threshold. Metadata of the video contains start time, end time, and point of interest Proposed algorithm will focus on maximizing the coverage and reduce the redundancy.

\subsection{Algorithm of Decision System}

Input : Metadata of all Videos of the event : metdataList Output : List of video to be fetched :

Process: 
1) Get overlapping videos

2) Remove overlapping videos from matadatList

3) Updated metaList from above step is Videos to be fetched

\subsection{Algorithm GetOverlappingVideo}

Input : Metadata List of all uploaded metadata

Output : Set of overlapping videos

Process:

// search for overlapping videos for each metadata

For each (Metadata md :MetadataList)

\{

For each (Metadata md1: MetadataList )

\{

If time of md includes md 1 and difference of poi is less than variation threshold

Then md1 is overlapping video of md

\}\}

Get Set of overlapping videos from overlapping video list of each metadata.

\subsection{Steps of POI calculation Get POI of video}

Input : Video V, Reference Image R

Output : Double POI

Process:

Get Frames of video with duration of $1 \mathrm{sec}$ : Bitmap[] frames

Double poi=0;

For(Bitmap frame: frames)

\{

Poi $=$ poi + getPOIOfImage $($ frame, $\mathrm{R})$

\}

Poi $=$ poi / frames.length

Get POI of Image

Input : Reference Image R, Frame f

Output : POI of image poi

Process:

1) Convert Bitmap image to Matrix

2) Resize Both matrix to Same size $(512,512)$

3) Convert matrices to Bitmap

4) Get Brisk Feature of both images

5) Get Descriptor of Brisk Feature : KeyPoints

6) Match descriptors and get matched keypoints

7) Get average of Horizontal distance between all matched points : POI of image.

\section{EXPERIMENTAL SETUP AND EVALUATION \\ 5.1 Parameters to Evaluate proposed system \\ 1) Response time of system after video request.}

Time required to play video in proposed system $\mathbf{T p}$ (video sharing with decision system) will be compared with existing system Te (On demand video sharing). Time required by existing system is completely depends on the availability of video uploader but for experimental purpose availability of uploader is assumed.

2) Redundancy of fetched videos on server

In existing system, no. of videos uploaded for particular event is depends on demand of the viewers. For e.g. If metadata of 10 videos of same event is uploaded to the server then if viewer demands all 10 videos then all 10 videos will be uploaded to the video and it may result into redundant data on server. It is assume that all submitted metadata videos may be uploaded to the server in existing system. Number of fetched videos in existing system is denoted as $\mathrm{NFe}$. In proposed system, only unique videos are uploaded to the server, therefore no of fetched videos depends on redundancy in submitted metadata. Number of fetched videos in proposed system is denoted as NFp.

\subsection{Experimental Setup}

\section{For proposed System}

Register one event to the system reference image with all other required details with time. Use multiple android smartphones to capture the system. While capturing the system capture some overlapping videos to check whether proposed system eliminates overlapping videos. After event, run decision system and check how many videos are uploaded to server NFp. After running decision system, one viewer will request to watch one video and at that time capture Tp.

\section{For Existing System}

Register event same as described in above section and capture the videos for the event with multiple smart phones. Viewer will demand all videos whose metadata is uploaded to the server therefore all videos need to uploaded to the server (NFe :Number of videos uploaded to server in existing system). Time required for response on video demand is recorded Te.

\subsection{Result}

\begin{tabular}{|l|l|l|}
\hline Parameter & Existing System & Proposed System \\
\hline Time (ms) & Above 1000 & $50-200$ \\
\hline No of videos & $5 \quad$ out of 5 & 3 out of 5 \\
\hline
\end{tabular}

\section{CONCLUSION}

This work addresses the problem of high latency time in On demand video sharing system and proposes an algorithm which will automatically decides the extraction of videos from registered users of the event. Decision of extraction of the video is done with two objectives. First is minimize server storage space and another is minimize redundancy of videos. From experiment results, proposed system shows its effectiveness and achieves objective of the work 
In the future scope Angle of video can be calculated and added to the metadata of video for more accurate results of redundancy.

\section{REFERENCES}

[1] Seshadri Padmanabha Venkatagiri, Mun Choon Chan, Wei Tsang Ooi, Jia Han Chiam "On Demand Retrieval of Crowdsourced Mobile Video" IEEE sensors journal vol.15,no 5,May 2015.

[2] X. Bao and R. Roy Choudhury, "MoVi: Mobile phone based video highlights via collaborative sensing," in Proc. 8th Int. Conf. Mobile Syst., Appl., Services (MobiSys), 2010.

[3] http://www.crowdoptic.com

[4] X. Liu, M. Corner, and P. Shenoy, "SEVA: Sensorenhanced video annotation," ACM Trans. Multimedia Comput., Commun., Appl., vol. 5, no. 3, Aug. 2009.

[5] S. Greenhill and S. Venkatesh, "Distributed query processing for mobile surveillance," in Proc. 15th Int. Conf. Multimedia (MM), 2007.
[6] S. A. Ay, R. Zimmermann, and S. Kim, "Relevance ranking in georeferenced video search," Multimedia Syst., vol. 16, no. 2,2010.

[7] J. Hao, S. H. Kim, S. A. Ay, and R. Zimmermann, "Energy-efficient mobile video management using smartphones," in Proc. 2nd Annu ACM Conf. Multimedia Syst. (MMSys), 2011.

[8] P. Simoens, Y. Xiao, P. Pillai, Z. Chen, K. Ha, and M. Satyanarayanan, "Scalable crowd-sourcing of video from mobile devices," in Proc. 11th Annu. Int. Conf. Mobile Syst., Appl., Services (MobiSys), 2013.

[9] P. Jain, J. Manweiler, A. Acharya, and K. Beaty, "FOCUS: Clustering crowdsourced videos by line-ofsight," in Proc. 11th ACM Conf. Embedded Netw. Sensor Syst. (SenSys), 2013.

[10] N. Snavely, S. M. Seitz, and R. Szeliski, "Modeling the world from internet photo collections," Int. J. Comput. Vis., vol. 80, no. 2 Nov. 2008 